\title{
Hypokalemic Periodic Paralysis Associated with Glue-Sniffing
}

\author{
A. Anitha ${ }^{1} \cdot$ Vinoth Selvaraj $^{1} \cdot$ Senthil Kumar Kandasamy ${ }^{1} \cdot$ Javed Ismail $^{1}$
}

Received: 11 September 2015 / Accepted: 16 December 2015 / Published online: 13 January 2016

(C) Dr. K C Chaudhuri Foundation 2016

To the Editor: Hypokalemic periodic paralysis, characterized by episodic, reversible weakness with hypokalemia is rare in children. Glue sniffing can rarely lead to hypokalemic periodic paralysis. We report a case of hypokalemic periodic paralysis precipitated by glue sniffing.

A 10-y-old boy presented with acute onset generalized weakness of all four limbs and floppiness of neck since past few hours. He had no sensory impairment, respiratory muscle weakness or bladder bowel disturbance. He had bradycardia (heart rate 55/min); ECG showing $\mathrm{U}$ waves and his serum potassium was $1.4 \mathrm{mEq} / \mathrm{L}$. Initial venous blood gas revealed high anion gap metabolic acidosis (Anion gap $=24$ ) which subsequently became normal. Serum potassium was corrected with intravenous supplementation and his weakness improved dramatically. On evaluation for cause of metabolic acidosis, his urine $\mathrm{pH}$ was 7 with hypercalciuria (calcium creatinine ratio of 2); fractional excretion of bicarbonate was $2.2 \%$ (after bicarbonate loading) and urine minus blood $\mathrm{CO}_{2}$ was 9 which were suggestive of distal renal tubular acidosis (RTA). Ultrasound KUB (kidney, urinary bladder) revealed bilateral medullary nephrocalcinosis. On detailed evaluation, there was no significant past or family history of similar illness but he revealed habit of glue sniffing with his friends for past $2 \mathrm{y}$. He was a school dropout and on evaluation by child psychiatrist, he was diagnosed to have Attention Deficit Hyperactivity Disorder (ADHD). He was started on pharmacotherapy and

Javed Ismail

javedisi86@gmail.com

1 Department of Pediatrics, Jawaharlal Institute of Postgraduate Medical Education and Research, Puducherry 6, India cognitive behavior therapy and was discharged on oral potassium and bicarbonate supplements. On follow up at 2, 4 and 8 wk, his acidosis and hypokalemia resolved and supplements were tapered and stopped.

Inhalant abuse is common but least studied form of substance abuse. In India, about $18.8 \%$ of school children have ever used inhalants and glue is the most commonly abused inhalant [1]. Risk factors identified in index case were ADHD, poor socioeconomic status, lack of relationship with parents and peer pressure. In a study by Mannuzza et al., about $50 \%$ of children with ADHD symptoms had a substance abuse disorder [2]. Children with ADHD tend to have substance abuse at an earlier age, longer duration of substance abuse, and may progress to alcohol or other drug abuse disorders [3]. Thus, early treatment of ADHD may prevent the development of substance abuse in adolescence.

Compliance with Ethical Standards

Conflict of Interest None.

Source of Funding None.

\section{References}

1. Akoijam BS, Jamir MN, Phesao E, Senjam GS. Inhalant use among schoolchildren in Northeast India: a preliminary study. Subst Abuse Res Treat. 2013;7:185-90.

2. Mannuzza S, Klein RG, Bessler A, Malloy P, LaPadula M. Adult outcome of hyperactive boys. Educational achievement, occupational rank, and psychiatric status. Arch Gen Psychiatry. 1993;50:56576.

3. Sullivan MA, Rudnik-Levin F. Attention deficit/hyperactivity disorder and substance abuse. Diagnostic and therapeutic considerations. Ann N Y Acad Sci. 2001;931:251-70. 